strategy can be adjusted. It is assumed that control of disease activity avoids or reduces signs and symptoms in the short term, and reduces the development of joint damage and disability in the long term.

Objectives To study if the decision-support system is effective in a) reducing disease activity, and b) maintaining the level of joint damage and disability in RA patients.

Methods 264 RA patients from 63 rheumatologists were included in a longitudinal evaluation, starting with a 3 month control period P0 (no feedback) followed by a 12 month intervention period P1 (with provision of feedback). Patients are nested in physicians, crossed with time nested in period. The main outcome variable is the Rheumatoid Arthritis Disease Activity Index (RADAI), a questionnaire on signs and symptoms in RA. The RADAI ranges from 0-10, higher scores indicate more disease activity. Disease activity (DAS28), disability (HAQ), and joint damage (X-ray score) were additionally measured in P1. To compare the development of the RADAI in P0 with P1, multilevel analysis (linear regression with random coefficients) was used; the unit of time is 30 days. For analysing differences in P1 for DAS28, HAQ and X-ray score, the paired t-test was used.

Results All patients had at least 1 feedback report at start of P1, $40 \%$ had $2,23 \%$ had 3-6 reports. The regression model shows no time effect for RADAI in P0: $-0.02(\mathrm{p}=0.75)$ and a small time effect in P1: -0.04 ( $p<0.0001$ ). The latter means ca. -0.5 RADAI point in 12 months. The difference between P0 and P1 is significant $(\mathrm{p}=0.0011)$. The time effects were not influenced by: age, gender, disease duration, baseline level of disease activity, number of consultations, or changes in DMARD therapy. Of the 202 patients on DMARD therapy during P1, $n=114$ had the same therapy at begin and end. Over P1, the DAS28 was reduced with $-0.4(1.2)$ points $(\mathrm{p}<0.0001)$, the HAQ remained stable with $-0.1(0.4)$ points $(\mathrm{p}=0.03)$, and the $\mathrm{X}$-ray score increased with $+6.4(10.4)$ points $(\mathrm{p}<0.0001)$.

Conclusion These results point to a significant but small effect of a decision-support system in reducing disease activity in RA. Over 12 months, the medication strategy was changed in only a minority of patients. The SCQM may increase its efforts to stimulate physicans to adjust treatment, e.g. by the implementation of explicit treatment guidelines. Other possible reasons for suboptimal effectiveness need to be explored, including limited treatment options and medication compliance.

\section{SAT0237 THE DEVELOPMENT AND VALIDATION OF A MARKOV MODEL FOR THE ECONOMIC EVALUATION OF (NEW) TREATMENTS IN RHEUMATOID ARTHRITIS}

${ }^{1} \mathrm{PM}$ Welsing, ${ }^{2} \mathrm{M}$ Hartman, ${ }^{1} \mathrm{AM}$ van Gestel, ${ }^{1} \mathrm{PL}$ Van Riel, ${ }^{2} \mathrm{JL}$ Severens, ${ }^{1} \mathrm{RF}$ Laan. ${ }^{\prime}$ Department of Rheumatology; ${ }^{2}$ Department of Medical Technology Assessment, University Medical Centre Nijmegen, Nijmegen, The Netherlands

10.1136/annrheumdis-2001.873

Background Due to the scarcity of resources (new) treatments need not only to proof that they are effective, but also cost effective. Especially in the case of new more costly treatment options as for rheumatoid arthritis, establishing the cost-effectiveness is relevant. Sometimes data about the costs and effects (in the long term) are absent, especially in the case of new treatment options. A method to overcome the problem of incomplete data and to extrapolate short-term endpoints to long-term outcomes is modelling.
Objectives To develop and validate a Markov model for the economic evaluation of (new) treatments in RA.

Methods A Markov model was developed with a cycle time of 3 months and a time-horizon of 5 years. In a Markov model patients are classified in a number of states (Markov states), defined by the severity of the disease. Development of the disease is defined as transitions between these states. Markov states were defined using the Disease Activity Score (DAS), and were valued in utility and medical and total costs using a dataset from a 48-week trial with methotrexate. The model was calculated for ?usual care? for patients in the first 5 years of their disease, using transition probabilities calculated from the 5 -years followup data of patients participating in an open prospective study of early (disease duration $<1$ year) RA. To test the validity of the model, the structure of the model, the inputs of the model, the results of the model, and the value of the model to the decisionmaker were assessed.

Results The costs and the utility showed a clear relation with the Markov states. The expected medical and total costs per patient over 5 years were DFL. 14.896, and DFL 27.889 respectively and the expected Quality Adjusted Life Years (QALY?s) were 3,27. Differences in patient characteristics like age, gender, disease duration, used DMARD?s, number of underwent orthopaedic procedures, and education had a moderate effect on the valuation of the Markov states in costs and utility. The input data for the Markov model was appropriate for the Dutch situation. The expected total costs seemed somewhat lower than the costs found in another Dutch study in the first six years of the disease, but the mean utility was comparable to utilities found in other studies. It appeared possible to validly extrapolate the course of the disease over 5 years with the Markov model, using transition probabilities calculated from only the first year of follow-up of the patients in the open study. In this study the value of the model for the decision-maker could not be well established.

Conclusion The developed Markov model is valid for use in economic evaluations in RA.

\section{SAT0238 THE COST OF OSTEOARTHRITIS AND ITS ASSOCIATION WITH THE SEVERITY OF THE DISEASE}

A Belisari, LG Mantovani. Centre of Pharmacoeconomics, University of Milan, Milan, ItalyBackground

10.1136/annrheumdis-2001.874

Objectives To assess the cost related to caring for Osteoarthritis (OA) patients in Italy and to verify any association between cost and severity expressed in terms of Lequesne Index (LI) score.

Methods The analytic framework of the present study was a prevalence-based Cost of Illness Analysis. The societal perspective was adopted with a 6 months time horizon. Subjects: Eleven rheumatologic centres were selected all over Italy. These recruited and interviewed the first 30 consecutive $\mathrm{OA}$ patients presenting to their office with a diagnosis of knee osteoarthritis. To this purpose, a questionnaire was developed so to collect information about: demography, concomitant localisation of OA, LI score, direct and indirect costs. The severity of the disease was assessed at the time of the visit. To investigate the association between cost and severity we used multivariate linear regression techniques.

Results A total of 277 patients were recruited and interviewed. The average age was $65,7( \pm 9,8)$ years, the average LI score 
was $12,3( \pm 4,5)$ and the $73 \%$ were female subjects. In 6 months the burden to society to care for an OA patient was around 1.993 Euro (year 2000 value). Almost 33\% of this cost was represented by direct costs and about $67 \%$ by indirect costs. In general, the most important component of cost was informal care, followed by productivity losses and direct medical cost. Statistical analysis: The total cost was significantly associated with disease severity ( $\mathrm{p}<0,001$ ), increasing with increasing severity as measured through LI quartiles.

Conclusion From our results, it appears that interventions reducing LI score are likely to reduce the amount of resources needed to care for OA patients.

\section{SAT0239 THE RESPONSIVENESS OF HEALTH STATUS MASURES AND UTILITY-BASED METHODS IN PATIENTS WITH RHEUMATOID ARTHRITIS}

${ }^{1} \mathrm{~F}$ Salaffi, ${ }^{1} \mathrm{~A}$ Stancati, ${ }^{2} \mathrm{M}$ Carotti, ${ }^{1} \mathrm{~W}$ Grassi. ${ }^{1}$ Department of Rheumatology, University of Ancona, Jesi, Italy; ${ }^{2}$ Department of Radiology, University of Ancona, Ancona, Italy

10.1136/annrheumdis-2001.875

\section{Background}

Objectives To compare the responsiveness of disease-specific (Arthritis Impact Measurement Scale 2, AIMS2), ${ }^{1,2}$ generic (Medical Outcome Study Short Form Health Survey, SF-36) ${ }^{3}$ and preference-based instruments (rating scale, RS and time trade off, TTO $)^{4}$ to changes in particular status and perceived health in patients with rheumatoid arthritis (RA).

Methods Seventy-eight consecutive patients with RA, attending the care facilities of the Department of Rheumatology of Ancona, were recruited to the longitudinal study. Changes in global disease activity assessed by using the EULAR criteria were used as external indicators of improvement/response. In order to assess the responsiveness three strategies were used: 1) effect size (ES, mean change/standard deviation of baseline values); 2) standardised response mean (SRM, mean change/standard deviation of change in stable subjects); and 3) receiver operating characteristic method.

Results There were 55 women and 23 men with a mean age of 56 years (range 19-78 years) and arthritis duration of 7.1 years (range 6 months-24 years). By using a 3 category EULAR criteria for improvement/response, $21(27 \%)$ patients reported a good improvement, $23(29,5 \%)$ moderate improvement, and 34 $(43,5 \%)$ no changes in their arthritis over 12 months. The mean change score in generic and specific health status instruments and in utility measures, were significantly related to response category. There were no significant differences in responsiveness between SF-36 and AIMS2 in patients with RA when using changes in global disease activity as external indicator. The pain subscales were most sensitive to measure change over time, followed by physical function and psychosocial subscales. For the utility measurement, RS scores were found to be more responsive in detecting significant changes in preferences than TTO scores.

Conclusion The results suggest that both the SF-36 and AIMS2 may prove suitable for the assessment of health perception outcomes in RA clinical trials. Concerning the patient utilities the RS method appeared to be more responsive than TTO method. New strategies for assessing sensitivity to small changes should be developed and applied to health status and utility measures.

\section{REFERENCES}

1 Meenan RF, Mason JK, Anderson JJ, Guccione AA, Kazis LE. AIMS2. The content and properties of a revised and expanded arthritis impact measurement scales health status questionnaire. Arthritis Rheum. 1992;35:1-10

2 Salaffi F, Piva S, Barreca C, on behalf of Gonarthrosis and Quality of Life (GOQUOLA) Study Group. Validation of an italian version of the Arthritis Impact Measurement Scales 2 (ITALIAN AIMS2) for patients with osteoarthiritis of the knee. Rheumatology 2000;39:720-6

3 Ware JE Jr, Sherbourne CD. The MOS 36-item short form health survey (SF-36). 1 Conceptual frame-work and item selection. Med Care 1992;30:473-81

4 Torrance G. Utility approach to measuring health related quality of life. J Chronic Dis. 1987;40:593-600

\section{SAT0240 COST EFFECTIVENESS OF CYCLOSPORINE (SANDIMMUNNEORAL ${ }^{\oplus}$ ) TREATMENT IN EARLY SEVERE RHEUMATOID ARTHRITIS: THE GRISAR STUDY}

${ }^{1}$ LG Mantovani, ${ }^{1} \mathrm{~A}$ Belisari, ${ }^{1} \mathrm{M}$ Pisani, ${ }^{2} \mathrm{G}$ Pasero, ${ }^{3} \mathrm{E}$ Marubini, ${ }^{4} \mathrm{~F}$ Priolo, ${ }^{5} \mathrm{R}$ Ferrara, $\mathrm{O}$ Della Casa Alberighi ${ }^{6} .{ }^{1}$ Center of Pharmacoeconomics; ${ }^{2}$ Institute of Rheumatology, University of Pisa, Pisa; ${ }^{3}$ Institute of Medical Statistics and Biometry, University of Milan, Milan; ${ }^{4}$ Institute of Radiology, Catholic University, Rome; ${ }^{5}$ Medical Department, Novartis Farma, Origgio, Italy; ${ }^{6}$ Clinical Research and Development, Novartis Pharma, Basel, Switzerland

\subsection{6/annrheumdis-2001.876}

Background Rheumatoid Arthritis (RA) is a chronic, degenerative, disabling and costly disease, particularly in its early form. There is evidence that cyclosporine A (CsA) is a disease modifying antirheumatic drug (DMARD) and is effective in slowing down the joint damage progression of early RA.

Objectives To assess the economic profile of CsA.

Methods TECHNIQUE: Incremental cost effectiveness analysis (CEA). PATIENTS AND DATA: collected from the GRISAR study, a multicenter, prospective, long-term, open-label with a blinded radiological end-point, randomised study of CsA vs conventional DMARDs. ALTERNATIVES: CsA vs conventional DMARDs. PERSPECTIVE: Italian National Health Service. COSTS: direct healthcare costs (drugs, diagnostics, hospitalisations for serious a dverse events -SAEs-) expressed in Euro 2000. EFFECTS: progression in the eroded joint count (PEJC) according to the Larsen-Dale method and reduction in working capacity due to RA. OTUCOME: Incremental cost effectiveness ratio $[$ ICERpejc $=($ Ccsa-Cdmards $) /($ PEJC csa-PEJC dmards $)]$.

Results 248 early severe RA patients (F/M 196/52; mean age $48.7+11.9$ yrs) were considered in this analysis. The mean duration of f-up was 3.9 years. Compared to conventional DMARDs, CsA patients showed higher costs for drugs (Euro 12340 vs $1300, \mathrm{p}<0.05$ ), SAE and diagnostics (Euro 2540 vs $2430, \mathrm{P}=$ $\mathrm{NS}$ ), and total cost (Euro 14880 vs $3730, \mathrm{p}<0.05)$. The progression of RA measured through PEJC was slower in CsA patients compared to conventional DMARDs (1.9 vs 3.2, p < $0.05)$, resulting in an ICER of Euro 8,570 per erosion avoided. Compared to conventinal DMARDs, less patients in the CsA group stopped working because of RA $(2.7 \%$ compared to 7.8\%).

Conclusion This is one of the first prospective CEAs based on a prospective, long-term, randomised clinical trial with a pragmatic design in early RA. Our estimates show that CsA incremental effectiveness is achieved at a reasonable incremental cost. When considered in the perspective of early severe RA, CsA has a favourable pharmacoeconomic profile since CsA. 